\title{
The Analysis of the Quasi-likelihood Algorithm of the Estimated Parameter of the Signal with Unknown Localization within the Observation Interval
}

\author{
Oleg V. Chernoyarov ${ }^{1,2, *}$, Andrey P. Trifonov ${ }^{2}$, Konstantin S. Kalashnikov ${ }^{2}$ and Boris I. Shakhtarin ${ }^{3}$ \\ ${ }^{1}$ National Research Institute "Moscow Power Engineering Institute", Krasnokazarmennaya st., 14, Moscow, 111250, Russia \\ ${ }^{2}$ Voronezh State University, 1, Universitetskaya sq., Voronezh, 394006, Russia \\ ${ }^{3}$ Bauman Moscow State University, 5, Baumanskaya 2-ya st., Moscow, 105005, Russia \\ ${ }^{*}$ Corresponding author
}

\begin{abstract}
We carried out the synthesis and analysis of the quasilikelihood estimation algorithm of regular parameter of an untailored pulse with the unknown moments of appearance and disappearance observed against additive Gaussian white noise. We studied the influence of prior ignorance of the moments of appearance and disappearance on accuracy of the introduced estimate. As an example we considered the quasi-likelihood estimates of the amplitude and initial phase of a rectangular radio signal. And we found the loss in estimation accuracy due to ignorance of the moments of appearance and disappearance.
\end{abstract}

Keywords-moments of appearance and disappearance; unknown parameter; quasi-likelihood estimate; bias; dispersion; signal-to-noise ratio; amplitude; initial phase

\section{INTRODUCTION}

In a number of works [1-3, etc.] there is considered the problem of reception of a signal with the unknown moments of appearance and disappearance. In particular, in [3] the maximum likelihood estimates of parameters of such a signal are studied. However, as a rule, the maximum likelihood estimation algorithms of multiple signal parameters (in the present case - not less than three ones) can be practically realized by complex measurers being multichannel on each of estimated parameters. The measurer organization can be significantly simplified using the quasi-likelihood approach for estimation of informative parameters [4] while the a priori unknown moments of appearance and disappearance of the signal are substituted for their some expected values. In this context, we consider the problem of the estimated parameter of a signal of the known form with the unknown moments of appearance $\kappa$ and disappearance $\chi$.

II. THE ESTIMATE OF THE INFORMATIVE PARAMETER OF A SigNAL WITH THE KNOWN MOMENTS OF APPEARANCE AND DISAPPEARANCE

We write down such a signal mathematically as

$$
s(t, \kappa, \chi, \theta)= \begin{cases}f(t, \theta), & \kappa \leq t \leq \chi, \\ 0, & t<\kappa, t>\chi,\end{cases}
$$

where $f(t, \theta)$ is the function describing the signal form, $\theta$ is the unknown regular [5] parameter to be estimated,

$$
\kappa \in\left[\kappa_{\min }, \kappa_{\max }\right], \quad \chi \in\left[\chi_{\min }, \chi_{\max }\right], \quad \kappa_{\max } \leq \chi_{\min }
$$

Let the signal (1) is received against additive Gaussian white noise $n(t)$ with one-sided spectral density $N_{0}$. Then, the realization of the random process is observed at the receiver input over the time interval $[0, T]$. Here $\kappa_{0}, \chi_{0}, \theta_{0}$ are the true values of the unknown parameters.

$$
x(t)=s\left(t, \kappa_{0}, \chi_{0}, \theta_{0}\right)+n(t)
$$

To synthesize the estimation algorithm of the informative parameter $\theta$ we use a maximum likelihood method $[5,6]$. We presuppose that the observation interval satisfies to a condition $0 \leq \kappa_{\min }<\chi_{\max } \leq T$ so the signal (1) is located completely within this interval. Besides, we take that $f\left(\kappa_{0}, \theta\right) \neq 0$, $f\left(\chi_{0}, \theta\right) \neq 0$, i.e. the signal (1) is discontinuous [5].

If the moments of appearance and disappearance are a priori known, then the logarithm of the functional of likelihood ratio (FLR) is written down in the kind of $[5,6]$

$$
L_{0}(\theta)=\frac{2}{N_{0}} \int_{0}^{T} x(t) s\left(t, \kappa_{0}, \chi_{0}, \theta\right) d t-\frac{1}{N_{0}} \int_{0}^{T} s^{2}\left(t, \kappa_{0}, \chi_{0}, \theta\right) d t
$$

As is widely known, the maximum likelihood measurer should generate the logarithm of FLR (4) and find the position of its absolute maximum in the prior domain of the parameter $\theta_{0}$. Therefore, the maximum likelihood estimate (MLE) $\theta_{m}$ of the parameter $\theta_{0}$ is determined by the expression

$$
\theta_{m}=\arg \sup L_{0}(\theta)
$$


Substituting Eq. (3) in Eq. (4) we present the logarithm of FLR as the sum of signal $S_{0}(\theta)$ and noise $N_{0}(\theta)$ components:

$$
L_{0}(\theta)=S_{0}(\theta)+N_{0}(\theta)
$$

where $S_{0}(\theta)=S_{0}\left(\theta, \theta_{0}\right)-Q_{0}(\theta) / 2$,

$$
\begin{gathered}
S_{0}\left(\theta, \theta_{0}\right)=\frac{2}{N_{0}} \int_{\kappa_{0}}^{\chi_{0}} f(t, \theta) f\left(t, \theta_{0}\right) d t \\
Q_{0}(\theta)=\frac{2}{N_{0}} \int_{\kappa_{0}}^{\chi_{0}} f^{2}(t, \theta) d t \quad N_{0}(\theta)=\frac{2}{N_{0}} \int_{\kappa_{0}}^{\chi_{0}} n(t) f(t, \theta) d t
\end{gathered}
$$

The noise component $N_{0}(\theta)$ is the Gaussian random process with zero mathematical expectation and correlation function $K\left(\theta_{1}, \theta_{2}\right)=\left\langle N_{0}\left(\theta_{1}\right) N_{0}\left(\theta_{2}\right)\right\rangle=S_{0}\left(\theta_{1}, \theta_{2}\right) \quad$ being identical in form to Eq. (7). Considering that the signal-to-noise ratio (SNR) $[5,6] z_{0}^{2}=Q_{0}\left(\theta_{0}\right)$ is sufficiently great, we pass to the normalized logarithm of FLR $L_{z}(\theta)=L_{0}(\theta) / z_{0}^{2}$ and then applying the method of small parameter as described in [6], we find the conditional bias and dispersion of MLE (5):

$$
\begin{gathered}
b\left(\theta_{m} \mid \theta_{0}\right)=\left\langle\theta_{m}-\theta_{0}\right\rangle=0 \\
D\left(\theta_{m} \mid \theta_{0}\right)=\left\langle\left(\theta_{m}-\left\langle\theta_{m}\right\rangle\right)^{2}\right\rangle=-\left[\left.\frac{d^{2} S_{0}(\theta)}{d \theta^{2}}\right|_{\theta=\theta_{0}}\right]^{-1}=\frac{1}{F\left(\kappa_{0}, \chi_{0}\right)}
\end{gathered}
$$

where

$$
F(\alpha, \beta)=\frac{2}{N_{0}} \int_{\alpha}^{\beta}\left[\frac{d f(t, \theta)}{d \theta}\right]_{\theta_{0}}^{2} d t
$$

III. THE EsTIMATE OF THE INFORMATIVE PARAMETER OF A SIGNAL WITH THE UNKNOWN MOMENTS OF APPEARANCE AND

\section{DISAPPEARANCE}

Now, let us assume that the moments of appearance and disappearance of a signal (1) is a priori unknown and there is no need to estimate them. In other words, the parameters $\kappa$ and $\chi$ are the spurious ones. According to $[5,6]$, one way to overcome the prior parametrical uncertainty is to find the MLEs of spurious parameters $\kappa_{m}, \chi_{m}$ and then to use these estimates instead of unknown values $\kappa_{0}, \chi_{0}$ in Eq. (4). Thus, the receiver should form the required estimate as

$$
\hat{\theta}_{m}=\arg \sup L_{m}(\theta), \quad L_{m}(\theta)=L\left(\kappa_{m}, \chi_{m}, \theta\right)=\sup _{\kappa, \chi} L(\kappa, \chi, \theta)
$$

where

$$
L(\kappa, \chi, \theta)=\frac{2}{N_{0}} \int_{0}^{T} x(t) s(t, \kappa, \chi, \theta) d t-\frac{1}{N_{0}} \int_{0}^{T} s^{2}(t, \kappa, \chi, \theta) d t
$$

Expression (12) defines the receiver organization, and, as follows from [3], under sufficiently great SNRs the characteristics of MLE (11) coincide with the characteristics (8), (9) of the estimate found at a priori known moments of appearance and disappearance. However, in this case it is necessary to form a three-dimensional random field (12) over all domain of prior values of parameters $\kappa, \chi, \theta$. Besides, the majority of parameters $\theta$ that need to be measured shall be found using the multichannel processing. The need for an estimation of two more spurious parameters leads to the additional significant increases in the number of channels, and, hence, to the considerable difficulties in receiver implementing.

A part of these difficulties is avoidable by using the quasilikelihood estimation algorithm [4]. In this case the receiver generates the logarithm of FLR (12) for some expected moments of appearance $\kappa^{*}$ and disappearance $\chi^{*}$ from the domain of prior values (2)

$$
\begin{gathered}
L_{q}(\theta)=L\left(\kappa^{*}, \chi^{*}, \theta\right)= \\
=\frac{2}{N_{0}} \int_{0}^{T} x(t) s\left(t, \kappa^{*}, \chi^{*}, \theta\right) d t-\frac{1}{N_{0}} \int_{0}^{T} s^{2}\left(t, \kappa^{*}, \chi^{*}, \theta\right) d t
\end{gathered}
$$

and it searches for the position of its maximum. As a result, we obtain the quasi-likelihood estimate (QLE) of the parameter $\theta_{0}$ of the kind of

$$
\theta_{q}=\arg \sup L_{q}(\theta)
$$

Let us find the accuracy characteristics of QLE (14). Substituting Eq. (3) in Eq. (13), similarly to Eq. (6), we have the representation $L_{q}(\theta)=S_{q}(\theta)+N_{q}(\theta)$, where

$$
S_{q}(\theta)=S_{q}\left(\theta, \theta_{0}\right)-Q_{q}(\theta) / 2
$$




$$
S_{q}\left(\theta, \theta_{0}\right)=\frac{2}{N_{0}} \int_{\max \left(\kappa_{0}, \kappa^{*}\right)}^{\min \left(\chi_{0}, \chi^{*}\right)} f(t, \theta) f\left(t, \theta_{0}\right) d t
$$

$$
Q_{q}(\theta)=\frac{2}{N_{0}} \int_{\kappa^{*}}^{\chi^{*}} f^{2}(t, \theta) d t \quad N_{q}(\theta)=\frac{2}{N_{0}} \int_{\kappa^{*}}^{\chi^{*}} n(t) f(t, \theta) d t
$$

The function $S_{q}(\theta)$ is the deterministic component at the receiver output. The noise component $N_{q}(\theta)$ is the Gaussian random process with zero mathematical expectation and correlation function

$$
K_{q}\left(\theta_{1}, \theta_{2}\right)=\frac{2}{N_{0}} \int_{\kappa^{*}}^{\chi^{*}} f\left(t, \theta_{1}\right) f\left(t, \theta_{2}\right) d t
$$

Let the absolute maximum of the function (15) is being made at some point $\theta^{*}$ that is

$$
\theta^{*}=\arg \sup S_{q}(\theta)
$$

We presuppose that SNR at the receiver output is sufficiently great so $z^{2}=S_{q}^{2}\left(\theta^{*}\right) / K_{q}\left(\theta^{*}, \theta^{*}\right)>>1$. In fulfilling the last condition we introduce the small parameter $\varepsilon=1 / z$ and pass to the normalized logarithm of FLR $L(\theta)=L_{q}(\theta) / S_{q}\left(\theta^{*}\right)$ which we present in the kind of $L(\theta)=S(\theta)+\varepsilon N(\theta)$. Here $S(\theta)=S_{q}(\theta) / S_{q}\left(\theta^{*}\right), N(\theta)=N_{q}(\theta) / \sqrt{K_{q}\left(\theta^{*}, \theta^{*}\right)}$ are the normalized signal and noise components. Then, the equation is to find QLE ${ }^{\theta_{q}}$ (14) can be written down as

$$
\left[d S_{q}(\theta) / d \theta+\varepsilon d N_{q}(\theta) / d \theta\right]_{\theta_{q}}=0
$$

We find the solution of Eq. (19) as series in terms of powers of $\varepsilon: \theta_{q}=\theta^{*}+\varepsilon \theta_{1}+\varepsilon^{2} \theta_{2}+\ldots$. Substituting the last expression in Eq. (19), expanding the function in square brackets into Taylor series in the neighborhood of point $\theta^{*}$ and limiting the first approximation we have

$$
\theta_{q}=\theta^{*}-\varepsilon\left[\frac{d N(\theta) / d \theta}{d^{2} S(\theta) / d \theta^{2}}\right]_{\theta^{*}}
$$

From here we obtain the conditional bias and dispersion of QLE in the form of

$$
b\left(\theta_{q} \mid \theta_{0}\right)=\theta^{*}-\theta_{0}
$$

$$
D\left(\theta_{q} \mid \theta_{0}\right)=\left[\frac{\partial^{2} K\left(\theta_{1}, \theta_{2}\right) / \partial \theta_{1} \partial \theta_{2}}{z^{2}\left(d^{2} S(\theta) / d \theta^{2}\right)^{2}}\right]_{\theta_{1}=\theta_{2}=\theta=\theta^{*}}
$$

where $K\left(\theta_{1}, \theta_{2}\right)=K_{q}\left(\theta_{1}, \theta_{2}\right) / K_{q}\left(\theta^{*}, \theta^{*}\right)$ is the correlation function of the normalized noise component.

We specify Eqs. (20) and (21) for some special cases.

\section{Some SPecial CASES OF THE QuASI-LIKELIHOOD ESTIMATE OF THE INFORMATIVE SIGNAL PARAMETER}

\section{A. The Shorter Reference Signal}

Let the values $\kappa^{*}$ and $\chi^{*}$ satisfy to the conditions

$$
\kappa^{*}>\kappa_{0}, \quad \chi^{*}<\chi_{0}
$$

so the received signal is more long than the expected (reference) signal. Then the limits of integration in Eq. (16) are $\max \left(\kappa_{0}, \kappa^{*}\right)=\kappa^{*}, \min \left(\chi_{0}, \chi^{*}\right)=\chi^{*}$. Using Eqs. (15), (16), from Eq. (18) we have $\theta^{*}=\theta_{0}$. Thus, according to Eq. (20), QLE (14) is unbiased. Further, substituting Eqs. (15) and (17) in Eq. (21) we obtain the expression for QLE dispersion in the kind of

$$
D\left(\theta_{q} \mid \theta_{0}\right)=1 / F\left(\kappa^{*}, \chi^{*}\right)
$$

where the function $F(\alpha, \beta)$ is defined in Eq. (10). Using Eqs. (9) and (23) we describe the loss in estimation efficiency due to the ignorance of the moments of appearance and disappearance by the ratio

$$
\rho=V\left(\theta_{q} \mid \theta_{0}\right) / D\left(\theta_{m} \mid \theta_{0}\right)=F\left(\kappa_{0}, \chi_{0}\right) / F\left(\kappa^{*}, \chi^{*}\right)
$$

Here $V\left(\theta_{q} \mid \theta_{0}\right)$ is the conditional variance of QLE (14) which coincides with its dispersion if the conditions (22) are satisfied.

\section{B. The Nonpower Informative Parameter}

Let the parameter $\theta$ is nonpower $[5,6]$, that is 


$$
\frac{d}{d \theta} \int_{\kappa^{*}}^{\chi^{*}} f^{2}(t, \theta) d t=0
$$

Taking into account Eq. (25), from Eq. (18) we get $\theta^{*}=\theta_{0}$. Thus, the position of the maximum of the signal component (15) coincides with true value $\theta_{0}$, and QLE (14) is unbiased. Further, substituting Eqs. (15), (17) in Eq. (21) and using Eq. (25) we find the conditional QLE (14) dispersion:

$$
D\left(\theta_{q} \mid \theta_{0}\right)=F\left(\kappa^{*}, \chi^{*}\right) / F^{2}\left[\max \left(\kappa_{0}, \kappa^{*}\right), \min \left(\chi_{0}, \chi^{*}\right)\right]
$$

Similarly to Eq. (24) we define the loss in accuracy of QLE (14) in comparison with MLE (5) as

$$
\rho=F\left(\kappa_{0}, \chi_{0}\right) F\left(\kappa^{*}, \chi^{*}\right) / F^{2}\left[\max \left(\kappa_{0}, \kappa^{*}\right), \min \left(\chi_{0}, \chi^{*}\right)\right]
$$

\section{Examples}

As an example we consider the estimate of the amplitude of a radio pulse with rectangular envelope when in Eq. (1) the function $f(t, \theta)=f(t, a)$ has the appearance

$$
f(t, a)=a \cos (\omega t-\varphi)
$$

With Eq. (28), from Eqs. (8) and (9) follows that MLE of the amplitude at the known moments of appearance and disappearance is unbiased and possesses the dispersion $D\left(a_{m} \mid a_{0}\right)=N_{0} /\left(\chi_{0}-\kappa_{0}\right)$

Substituting Eq. (28) in Eq. (16), and then Eq. (16) in Eq. (15), Eq. (15) in Eqs. (18), (20) and (21) we find the QLE bias and dispersion:

$$
\begin{gathered}
b\left(a_{q} \mid a_{0}\right)=a_{0}[\min (0, \delta \kappa)-\max (0, \delta \chi)] /\left(\chi_{0}-\kappa_{0}\right)(1-\delta \kappa+\delta \chi) \\
D\left(a_{q} \mid a_{0}\right)=N_{0} /\left(\chi_{0}-\kappa_{0}\right)(1-\delta \kappa+\delta \chi)
\end{gathered}
$$

where $\delta \kappa=\left(\kappa^{*}-\kappa_{0}\right) /\left(\chi_{0}-\kappa_{0}\right), \delta \chi=\left(\chi^{*}-\chi_{0}\right) /\left(\chi_{0}-\kappa_{0}\right)$. As before, we write down the loss in accuracy of QLE (14) of the amplitude in comparison with corresponding MLE (5) like that

$$
\rho=\frac{1}{1-\delta \kappa+\delta \chi}+z^{2}\left[\frac{\min (0, \delta \kappa)-\max (0, \delta \chi)}{1-\delta \kappa+\delta \chi}\right]^{2}
$$

In Fig. 1a the dependences of loss $\rho$ from the relative detuning $\delta \chi$ on the moment of disappearance are presented at fixed $\delta \kappa$ and SNR $z=10$. The curve 1 corresponds to $\delta \kappa=0$, that is the signal moment of appearance coincides with the expected $\kappa_{0}=\kappa^{*}$. Under $\kappa_{0} \neq \kappa^{*}$ the loss in accuracy of QLE increases both for positive $\delta \kappa=0.3$ (curve 2) and negative $\delta \kappa=-0.3$ (curve 3) detunings. As can be seen, more disadvantaged conditions of reception occur, if $\delta \kappa>0$ and $\delta \chi<0$. This is equivalent to the satisfaction of inequalities (22) when the signal appears earlier and disappears later than the expected signal. In Fig. $1 \mathrm{~b}$ the dependences of loss in QLE accuracy of the amplitude from the relative detuning $\delta \kappa$ on the moment of appearance are shown for cases of various $\delta \chi$ : $\delta \chi=0$ (curve 1), $\delta \chi=0.3$ (curve 2), $\delta \chi=-0.3$ (curve 3). It follows that the larger losses are realized when the inequalities (22) hold.

Let the estimated parameter is the initial phase of a rectangular radio pulse (28). Using Eqs. (8), (9), (26) we obtain that MLE and QLE of the initial phase are unbiased and they have conditional dispersions respectively. From here the loss in QLE efficiency (27) is

$$
\begin{gathered}
D\left(\varphi_{m} \mid \varphi_{0}\right)=N_{0} / a_{0}^{2}\left(\chi_{0}-\kappa_{0}\right) \\
N_{0}(1-\delta \kappa+\delta \chi)
\end{gathered}
$$

$\rho=(1-\delta \kappa+\delta \chi) /[1+\min (0, \delta \chi)-\max (0, \delta \kappa)]^{2}$

In Figs. 2 the dependences of loss (29) are drawn from relative detunings on the moment of disappearance $\delta \chi$ and the moment of appearance $\delta \kappa$. In Fig. 2a curve 1 corresponds to $\delta \kappa=0,2-\delta \kappa=0.3,3-\delta \kappa=-0.3$. In Fig. 2b curve 1 is calculated with $\delta \chi=0,2-\delta \chi=0.3,3-\delta \chi=-0.3$.

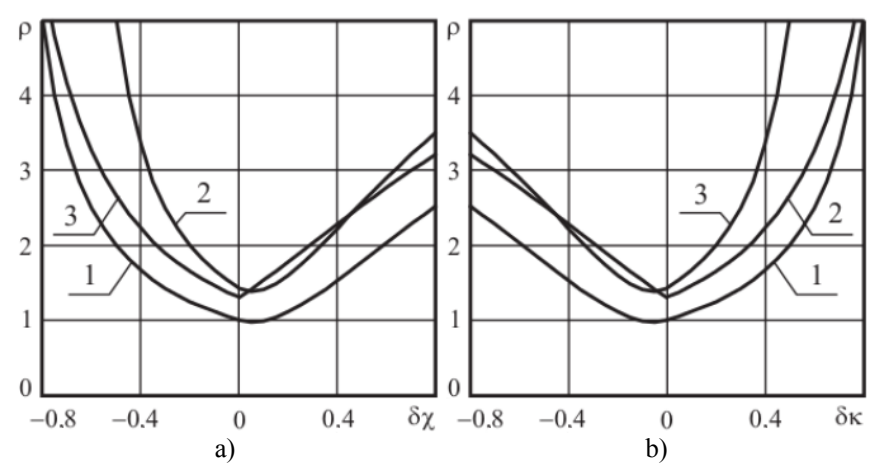

FIGURE I. THE LOSS IN EFFICIENCY OF THE AMPLITUDE ESTIMATE DUE TO THE INACCURATE TIME SIGNAL LOCALIZATION

As can be seen from the considered examples, when using QLE the losses in efficiency (24), (27) can be sufficiently great. It means that the ignorance of the signal moments of appearance and disappearance can lead to the significant decrease in the quality of an estimation of informative parameter. One way to overcoming the specified disadvantage 
of QLE is to carry out the simultaneous estimation of all unknown (both informative and spurious) parameters [4] as, for example, it is made in Eq. (11).

The findings allow us to permit judicious selection of the estimation algorithm depending on the requirements for the quality of the estimate and for the ease of technical implementation of the receiver.

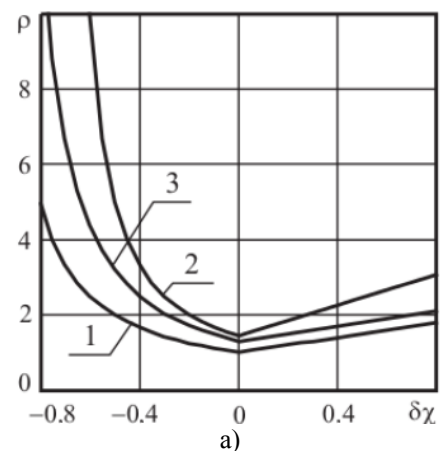

a)

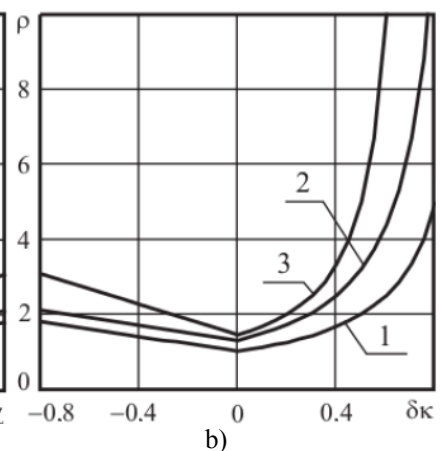

b)

FIGURE II. THE LOSS IN EFFICIENCY OF THE INITIAL PHASE ESTIMATE DUE TO THE INACCURATE TIME SIGNAL LOCALIZATION

\section{CONCLUSION}

The inaccurate signal localization within the observation interval can lead to the essential decrease in quality of the estimate of informative parameter. The detunings on the moments of appearances and disappearances are the least preferred when the reference signal duration less than received signal duration is. The accuracy of estimate of the informative parameter can be increased by simultaneous estimation of it and signal localization parameters within the observation interval, but the receiver organization is also complicating.

\section{REFERENCES}

[1] O.V. Chernoyarov, E.V. Chernoyarova, D.N. Shepelev, "Evaluation of dispersion and time parameters of a random radio-impulse at the background of a white noise" [in Russian], St. Petersburg State Polytechnical University Journal.Computer Science. Telecommunications and Control Systems, no. 4-1(52), pp. 122-127, December 2007.

[2] A.P. Trifonov, Yu.E. Korchagin, "Exact formulas for computation of reception characteristics of a signal with unknown appearance and disappearance times", Problems of Information Transmission, vol. 45, no. 2, pp. 91-100, February 2009.

[3] A.P. Trifonov, Yu.E. Korchagin, "Joint parameter estimations of signal with unknown moments of appearance and disappearance". Izvestiya Vysshikh Uchebnykh Zavedenij. Radioelektronika, vol. 43, no. 5, pp. 34-43, May 2000.

[4] V.I. Mudrov, V.L. Kushko Measurement processing methods. Quasilikelihood estimates [in Russian]. Radio i Svyaz', Moscow, 1983.

[5] A.P. Trifonov, Yu.S. Shinakov, Joint discrimination of signals and estimation of their parameters against background [in Russian], Radio i Svyaz', Moscow, 1986.

[6] E.I. Kulikov, A.P. Trifonov, Signal parameter estimation against hindrances [in Russian]. Sovetskoe Radio, Moscow, 1978. 